\title{
The review of human-robot interaction system based on visual servo
}

\author{
Xia yifei \\ College of Mechanical Engineering and Automation \\ Xihua University \\ Chengdu, $\mathrm{CHN}$ \\ email:291030179@qq.com \\ Liu Jianxin \\ College of Mechanical Engineering and Automation \\ Xihua University \\ Chengdu, CHN \\ email:897331849@qq.com
}

\author{
Chen Fufu \\ College of Mechanical Engineering and Automation \\ Xihua University \\ Chengdu,CHN \\ email: 510509936@qq.com \\ Huang Kecheng \\ College of Mechanical Engineering and Automation \\ Xihua University \\ Chengdu,CHN \\ email: 1094928030@qq.com
}

\begin{abstract}
From the development of robot technology, the implementation of the intelligent, safe and friendly mobile robot has been a goal by the human. In this paper, the definition of physical human-robot interaction is introduced; The classical visual servo system is analyzed, and we conducted a comparison and analysis of the nonlinear relationship between image featur01es and the robot pose based on the classical PID controll, fuzzy control and the PI $\lambda D \mu$ controller which used to manage the nonlinear of visual servo system. In the end, we summarized the security strategy and the research progress, problems and challenges of physical human- robot interaction in this field in the future.
\end{abstract}

Keywords-Physical human-robot interaction; Visual servoing; PIגD $\mu$ controller; Safe strategy

\section{INTRODUCTION}

In recent years, with the industrial robot technology becoming more mature, robot research hotspot has gradually shifted to the intelligent robot, and the visualcontrol is an important research direction at present. We generally use the industrial robot teaching or off-line programming method for machining task of path planning and motion programming for the industrial robot, so the robot only can perform mechanical work based on the program, when the environment changes, the intelligence of robot is too poor to change independently movement patterns, that will affect seriously the processing quality and efficiency. In order to improve the application range of machining efficiency and quality and expand the scope of application of robot, we want to improve the intelligence of the robot,which can get rid of the isolation strip and achieve real-time human-computer interaction together with the operator, but these have brought new challenges for the robot research field,namely in order to ensure the security of physical people, which requires that security strategy is very perfect,when the operator and robot work cooperatively.Because of the robot work space environment and the constraints, the control strategy is difficult to directly use the signals collected by the visual system,so Safety control strategy is a difficulty for the physical humanrobot interaction. The combination of image processing and control technology make visual servo robot become a very active subject, due to the control of robot vision system image signal acquisition and robot pose is not a simple linear relationship, so the design of the controller must ensure real-time in processing a large amount of image information, which can timely command and plan the trajectory of robot, at the same time what algorithm is used to realize image information into robots movement adjustment strategy is very important[1] [2] [9] [10].

Now, many researches have been carried out in all aspects of human-robot interaction system based on visual servo, and put forward some important solutions. Therefore,we reviewed physical research work in the field of human-computer interaction from the visual servo, the safe strategy of human-robot interaction and visual servo control algorithm, and pointed out that the challenges and development directions of the human-robot interaction system based on visual servo.

\section{VISUAL SERVO SYSTEM}

The servo control of the robot by using visual information is called visual servo. Visual servo is a visual control, whose information is used for feedback signal in visual control. The traditional visual servo system can be divided into two categories:the position based visual servo (PBVS) and the image based visual servoing (IBVS) [4] [8].

The position based visual servo (PBVS) uses the position signal collected by cameras as the given robot system. The control block diagram is shown in Figure 1 . 


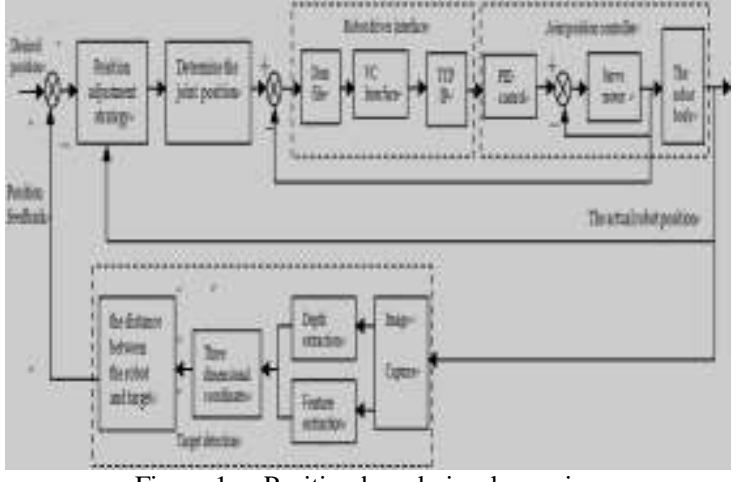

Figure 1. Position based visual servoing

The Visual Servo System is the Eye-in-hand structure, namely the camera is placed in the end of the robot. In order to obtain Cartesian coordinates of the 3D object and the distance of the object and the target robot tool, we conduct the digital image processing of the depth and feature extraction by the camera capturing image information of object and the robot,then make a comparison between actual distance and the expected position. Finally, we carry out position adjustment for position deviation by the position adjustment strategy. According to the deviation of each joint of the robot pose and expected pose, we carry out pose adjustment by the joint position strategy, who is to limit robot end position in a certain range for protecting the robot and operators, at the same time, avoids the singular pose.Because the control belogs to Looking and Doing way, so the real-time requirement is very high for the position adjustment strategy, the robot drive interface and joint position controller . Otherwise, it will affect the control precision and the safety of operators. Compared with the IBVS, the error signal has clear physical meaning, and PBVS was easier to implement. But because it is not directly on the image control,the kinematic error of the robot and The calibration error of the camera have a direct impact on the control accuracy of the system.

The image based visual servoing (IBVS) use directly of image features for robot control. the image feature of the target is given by the robot controller, it using the feature of the target image that is measured by the cameras regards as feedback, then we use the image deviation to control the robot's movement.The control block diagram is shown in Figure 2.

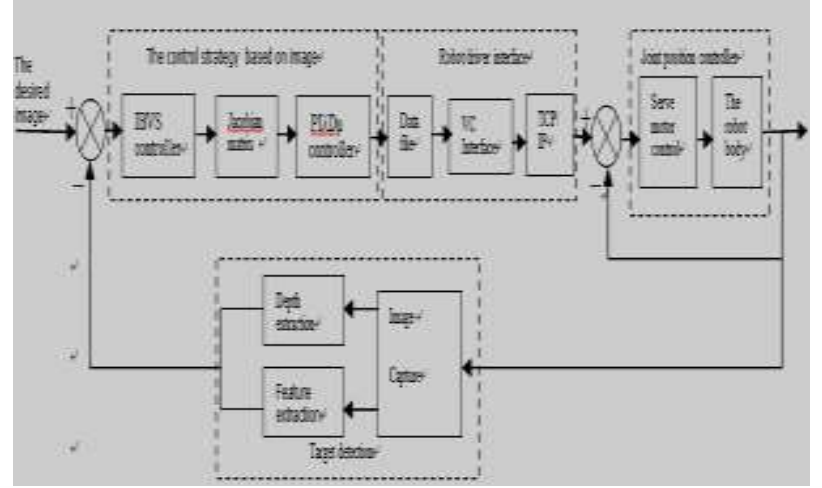

Figure 2. Image based visual servoing
The characteristics of IBVS:

1) The system is not sensitive to the calibration error and model error, and have a high control precision, but the error signal is the image feature, has no real physical meaning;

2) The image Jacobian matrix is a main task and problem for IBVS. There are three kinds of solving methods in common use: the direct estimation method, depth estimation method and constant approximation method,but Bthe they are difficult to solve.

3) The position of the robot end and the image characteristics is a nonlinear relationship, so the design of the control strategy based on IBVS will become very complicated. The traditional PID control is proposed for linear systems, so only can guarantee the stability of the system in a small scope,but to deal with the nonlinear system of fractional order PID control provides a new way for us, we will carry on the detailed in the fourth section.

\section{SAFE STRATEGY}

Traditional security concept of Physical humanrobot interaction interaction is through setting isolation belt for the robot, where does not allowoperators to enter,because it didn't really realize the human-robot interaction, so it can not meet the industrial requirements now. Now we need to design a complete strategy of human- robot interaction in the human "zero distance contact" at the same time to ensure the safety of the operators and the robot itself. Interactive challenges not only detect the collision in real-time, but also how to use visual closed loop in the execution of a robot to avoid collision.

In recent years, researchers have carried out extensive research in all aspects of the Physical humanrobot interaction, and put forward some solutions. there are two methods commonly to use in the safety monitoring and Protecting the physical person, robot collaborative work environment, One is based on visual method, such as we analyze the gesture movement, color, and texture through the visual system collecting signals;Another method is based on the inertial sensor,although its real-time performance is good, yet because the sensors placed in the robot,So it cannot monitor the changes in the surrounding environment, a moving object may hurt the operator. The Human-robot interaction safe strategy includes two parts: the collision detection strategy and collision avoidance strategy.

\section{A. The collision detection strategy}

Effective collision detection based on vision is for many years has been become the focus of experts. Ebert put forward a method of emergency stop to avoid collision by using an aspecial vision chip;Gecks and Henrich detected obstacles by using a multi camera system.However, the movement of objects may occur at any time in the surveillance area, which may lead to the visual system appear inconsistent with previous color and texture, and the illumination change will affect the whole 
precision and efficiency of collision detection. As a result, the whole area within the scope of the image depth information must have a very good robustness for the change of color and light. Therefore, Fischer and Henrich proposed a method for collision detection by using multiple 3D depth image; Schiavi proposed a method to realize the collision detection by using a ToF camera[13]. Some of these methods, the security area is static and cannot be changed. Therefore, it can't achieve real people in close collaboration with robot, and affects the work efficiency. So developing a real-time safe solution that is cost-effective for dynamic manufacturing environment becomes very necessary, the following is a new way to study recently, its block diagram shown in figure 3.

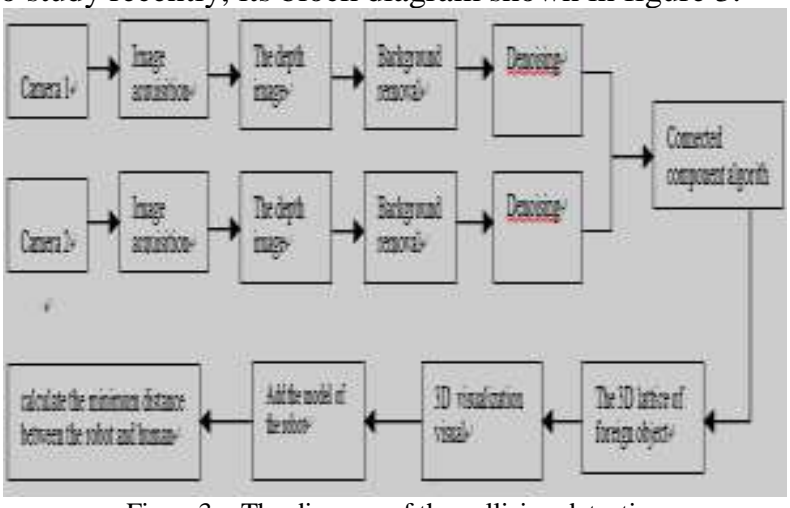

Figure3. The diagram of the collision detection

The system adopts the binocular vision system, the depth image of the human and the robot through the cameras. We remove the background through the adjacent frame difference method, after the background removal,only the foreign objects are left in the depth images, then apply a noise-removal filter and image enhancement processing. In order to get external object's 3D image information, We will have two cameras to obtain foreign object lattice information merge into a 3D dot matrix image information by a connected component algorithm. Now we have a complete lattice image of foreign matter and robot 3D, which can calculate the minimum distance of the foreign objects with the robot, that provides a basis for the collision avoidance strategy that we adopt.

\section{B. The collision avoidance strategy}

The collision avoidance strategy generally has two methods: The first is to stop the robot movement when the close proximity of an operator has been detected, and continue the robot movement after the operator walked away, moreover, it influences the efficiency of the robot. The second strategy is applicable to such operations as transportation where the path can be dynamically modified to avoid collision with human operators and other obstacles. Apparently, the second method realizes the real-time interaction of the robot and operating personnels, and does not need to stop the robot, the robot changes trajectory in real time through the distance of the end of robot and foreign obstacles, the specific principle diagram as shown in figure 4 .

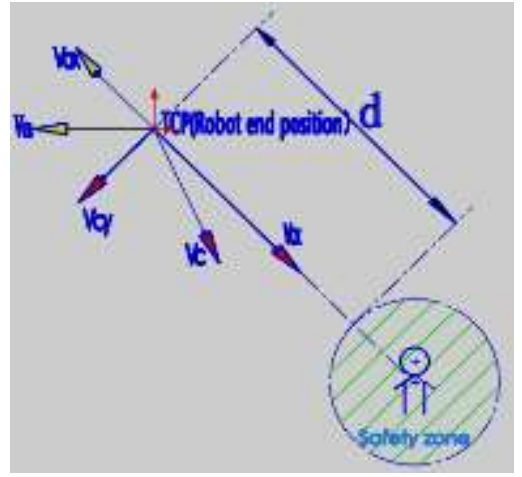

Figure 4. The control structure of fractional order PID controller

When the robot towards the operating personnel, the moving speed $\mathrm{Vc}$ is decomposed into parallel to the operator's velocity $\mathrm{Vcx}$ and perpendicular to his velocity Vcy. When the safety distance from the robot is less than d, the robot moves towards the reverse motion parallel to the operator; When the distance is greater than the d, we don't change its trajectory and make it continue along $\mathrm{Vc}$ movement. Because the computer always plans its trajectory, so the processing of data is very large, and then the real-time requirement is very high.

\section{THE PID CONTROLLER}

With the rapid development of the computer, the fractional order $\mathrm{PI}^{\lambda} \mathrm{D}^{\mu}$ control as a new engineering tool has attracted extensive attention of many domestic and foreignresearchers in recent years. And the practical application of the image processing in the field has achieved good results, including image enhancement,image denoising, image edge detection, image segmentation and image singularity detection and so on[5] [6].

The traditional PID controller made full use of the information of the present(P), the past (I) and future (D), And when the sampling period is small, the digital implementation is feasible. The PID controller is the main advantage that is to design the controller to eliminate the errors by the the error of output value and the expected value. And it does not depend on the Input - output model of the object,so it is widely used in the uncertain object model in actual engineering. But due to describing the camera model of the relationship between image characteristics and the robot end position is a nonlinear relationship, PID control algorithm can only guarantee the stability of the system in a small range for this, We have to control the limiting its input for the stability of the system, which is restricted to the pose of robot motion, so the traditional PID is often difficult to achieve satisfactory control effect[7].The Fuzzy control has the ability of learning andestimation for The unknown function and doesn't depends on the accurate mathematical model of objects, which is Suitable for strong coupling nonlinear system. But it must depend on the rule base and the human experience, so visual servo control based on image by The fuzzy control also brings certain difficulty. 
Compared to the traditional PID control and fuzzy control, the fractional order PID controller has more stable global scope. It is a new type of controller and generalized to the fractional domain, which Inherits the advantages of traditional PID and has the ability to deal with nonlinear systems and effectively overcome the shortcomings. These can greatly improve the quality of image based visual servo control, the controller has the following advantages:

1) It has greater flexibility. Due to the increase of the integration order and differential order, the scope of its choice than the conventional PID controller can be bigger .

2) It is more robust.Because it is insensitive to the changes of the control parameters and system parameter of the controlled object, As long as the parameters vary within a certain range, it can be a very good control.

3) Due to the differential item has the memory of past events, so the control precision is better guaranteed.

The fractional order PI $\lambda \mathrm{D} \mu$ controller is the extension of the traditional PID controller, the control structure is shown in figure 5:

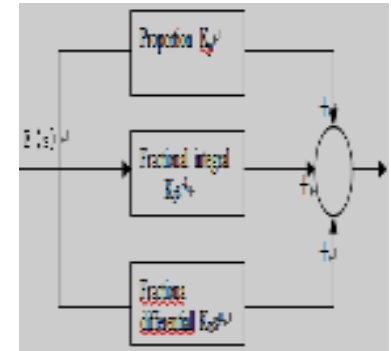

Figure 5. Collision avoidance strategy

\section{CONCLUSION}

When the physical man appeared in the robot working space,the safety and reliability of the robot is put forward the great demand, the safety of the people is the precondition of the robot working at this time. It brings a big challenge in robotics, this paper summarizes two kinds of traditional visual servo system, we Put forward the viewpoint that the fractional order PID controller for the nonlinear characteristics of the visual servo system based on image; At the same time, we analyzed the collision detection and collision avoidance safety strategy in detail,and proposed a new collision avoidance strategy. These results greatly enhance flexible man-machine collaboration, at the same time, in the case of getting rid of the barriers it ensure the absolute safety of operating personnel, all of which make the robot's productivity greatly increased. Our future work will focus on how to develop and improve the function of collision strategy that makes people for more complex operations tasks.

\section{ACKNOWLEDGEMENT}

I would like to acknowledge the support from Postgraduate Innovation Fund of Xihua University (ycjj2014059), the university key laboratory of Manufacturing and Automation of Sichuan Province at Xihua University and the support from the Professor Liu Jianxin for the second author.

\section{REFERENCES}

[1] Wu Hai-bin, Yang Jian-ming. The security research progress in the process of robot in human-robot interaction [J]. Chinese Safety Science Journal,2011,21(11):79-84

[2] Xiong Gen-liang,Chen hai-chu. Research and development status of physicalhuman- robot interaction [J]. Optics and Precision Engineering,2013,21(2):356-369

[3] Luo Ming,Wu Huai-yu,Du Zhao-jun. Research on mobile robot navigation based on human-robot interaction [J]. Computer Measurement and Control,2013,21(5):1291-1293

[4] Li Shiyong.Vision measurement and control for robots [M].BeiJing:National Defense Industry Press,2010.10

[5] Qing Chang-mao. Research on fractional order PID control and adrc for hypersonic vehicle [D]. Harbin .Harbin Institute of Technology,2011

[6] Huang Guo,Xu Li,Pu Yi-fei. Review of research in image processing of fractional calculus [J]. Computer Application Research,2012,29(2):

[7] Wang Zhenbin, Cao Gunagyi, Zeng Qingyi ,Zhu xinjian]. FractiOnaI order PID controllerr and its digtal implementation [J] Journal of Shanghai Jiao Tong University,2004,38(4):516-520

[8] Cosmin Copota,Adrian Burlacub,Clara M.Ionescua,Corneliu Lazarb,Robin De Keysera.A fractional order control strategy for visual servoing systems [J].Mechatronics,2013,23( 2013 ):848-855

[9] Minoru HASHIMOTO. Control for Physical Human-robot Interaction Based on Online Update of Dynamics [J]. IEEE,2011,21(11):179-184

[10] Jiun-Yih Kuan;Tz-Hao Huang;Han-Pang Huang;Yen-Tsung Chen.Adaptive coupled elastic actuator developed for physical human-robot interaction[J].Advanced Robotics,2011,25(11/12):79-87

[11] Ren-Jeng Wang;Han-Pang Huang. Mechanically stiffness adjustable actuator using a leaf spring for safe physical humanrobot interaction[J]. Mechanika,2012, (1):244-257

[12] Ikemoto,Shuhei;Ben Amor, Heni; Minato,Takashi;Physical Human-Robot Interaction Mutual Learning and Adaptation [J].IEEE ROBOTICS AUTOMATION MAGAZINE,2012,19(4):24-35

[13] Wang, Ren-Jeng; Huang, Han-Pang. Contact-less and Programming-less Human-Robot Collaboration $[\mathrm{J}]$. Procedia CIRP,2013,7( 2013 ):545-550 